
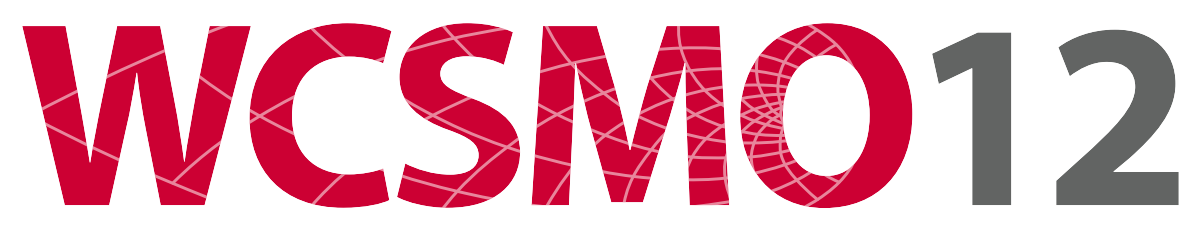

12th World Congress of Structural and Multidisciplinary Optimisation

Book of Abstracts 


\section{Gold Sponsors}
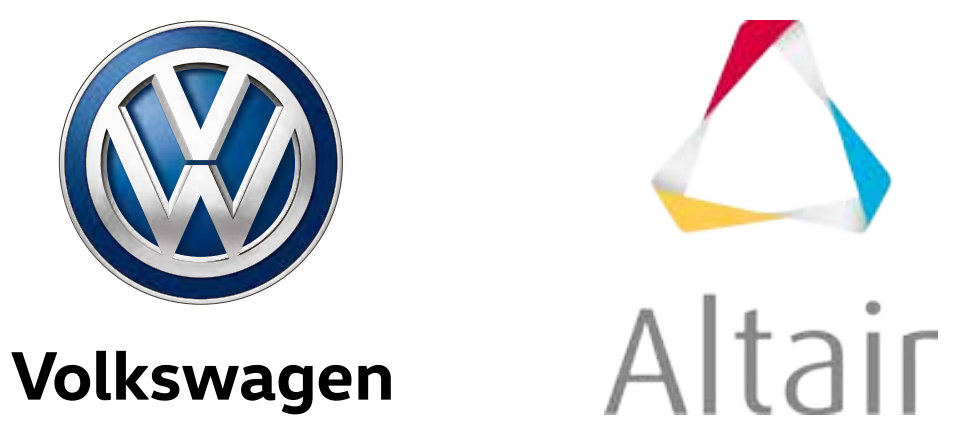

Silver Sponsors
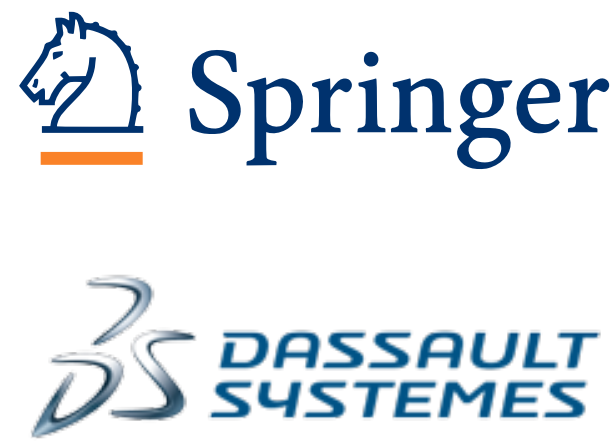

We also express our gratitude to the Deutsche Forschungsgemeinschaft (DFG) for their support.

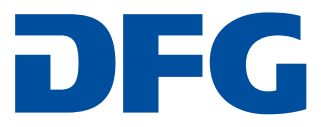

Deutsche

Forschungsgemeinschaft 
effect is through multilayered composites of a ferroelectric and a ferromagnetic material [1]. An applied electric field creates a piezoelectric strain in the ferroelectric, which produces a corresponding strain in the ferromagnetic material and a subsequent change in magnetization. Various efforts to improve the value of ME coupling coefficient have been made by modifying preparation techniques of the samples, by the proper choice of materials or different structures and by choosing different thickness of the samples [1]. In this study, we have applied a stochastic optimization for arriving at the solution for maximum "ME coupling coefficient $\alpha$ " of a laminar ME composite with the thickness and orientation of phases as design variables. As $\alpha$ could not be expressed explicitly as function of design variables, we have used the simulated annealing combined with a generalized Monte Carlo scheme for optimization problem. Optimal microstructure with single and poly-crystalline configurations that enhances the overall $\alpha$ is identified.

[1] Ortega et al., J.Phys.:Cond. Matter, 27,504002 (2015)

[2] Nan et al., Adv. Mater., 23, 1521 (2011)

\section{Generating the Best Stacking Sequence Table for the Design of Blended Composite Structures}

\section{F. Farzan Nasab, H. J. M. Geijselaers, I. Baran, A. de Boer}

In order to improve the ability of a large scale composite structure to carry tensile or compressive loads, stiffeners are added to the structure. The stiffeners divide the structure into several smaller panels. For a composite structure to be manufacturable, it is necessary that plies are continuous in multiple adjacent panels. To be able to prescribe a manufacturable design, an optimization algorithm can be coupled with a reference table for the stacking sequences (SST). As long as the ply stacks are selected from the SST, it is guaranteed that the design is manufacturable and all strength related guidelines associated with the design of composite structures are satisfied. An SST is made only based on strength related guidelines. Therefore, there exist a large number of possibilities for SSTs. Minimized mass is a typical goal in the design of aircraft structures. Different SSTs result in different values for the minimized mass. Thus it is crucial to perform optimization based on the SST which results in the lowest mass. This paper aims to introduce an approach to generate a unique SST resulting in the lowest mass. The proposed method is applied to the optimization problem of a stiffened composite structure resembling the skin of an aircraft wing box.

\section{Bootstrap guided information criterion for reliability analysis using small sample size information}

\section{Eshan Amalnerkar, Jihoon Kim, Woochul Lim, Tae Hee Lee}

Several methods for reliability analysis have been established and applied to engineering fields bearing in mind uncertainties as a major contributing factor. Small sample size based reliability analysis can be very beneficial when rising uncertainty from statistics of interest such as mean and standard deviation are considered. Model selection and evaluation methods like Akaike information criteria (AIC) have demonstrated efficient output for reliability analysis. However, information criterion based on maximum likelihood can provide better model 